\title{
IgG and IgM western blot assay for diagnosis of congenital toxoplasmosis
}

\author{
Anderson S Machado1, Gláucia MQ Andrade², José N Januário², Matheus D Fernandes', \\ Ana Carolina AV Carneiro', Mariângela Carneiro', Ericka VM Carellos², Roberta MC Romanelli², \\ Daniel V Vasconcelos-Santos ${ }^{2}$, Ricardo WA Vitor ${ }^{1 /+}$
}

${ }^{1}$ Departamento de Parasitologia, Instituto de Ciências Biológicas ${ }^{2}$ Faculdade de Medicina, Universidade Federal de Minas Gerais, Av. Antonio Carlos 6627, 31270-901 Belo Horizonte, MG, Brasil

\begin{abstract}
The aim of this study was to evaluate the utility of western blot (WB) analysis as a diagnostic tool for congenital toxoplasmosis in 215 newborn infants. The children were submitted to clinical examinations to assess macular, neurological and hearing signals. The WB results obtained were compared to the persistence of IgG antibodies at the end of 12 months, which is regarded as the "gold standard" diagnosis of congenital toxoplasmosis. Association between the WB results and the clinical signs presented by the infants was also assessed. Of the 215 children, 177 had a confirmed congenital toxoplasmosis diagnosis and 38 were uninfected. IgG-WB showed a sensitivity of $73.5 \%$ and a specificity of $97.4 \%$. IgM-WB showed a sensitivity of $54.8 \%$ and a specificity of $94.7 \%$. The IgG-WB and IgMWB combination increased the sensitivity to $86.5 \%$. The IgM-WB-positive children had a 1.4-fold greater risk of presenting active macular lesions than did those that were IgM-WB-negative. This study showed that the WB assay is a useful tool to confirm a diagnosis of congenital toxoplasmosis and that the IgM-WB-positive results can indicate active macular lesions in newborn infants.
\end{abstract}

Key words: congenital toxoplasmosis - western blot - macular toxoplasmosis

Primary Toxoplasma gondii infection of pregnant women can lead to congenital infection of the foetus. Most infected children do not present characteristic symptoms of the infection at birth, but there is a great risk of severe sequelae occurring during the first year of life or during infancy (Gilbert \& Peckham 2002). The absence of diagnosis at birth and consequent delay in treatment can predispose the infected child to macular, hearing and neurological symptoms (Andrade et al. 2008, Vasconcelos-Santos et al. 2009). Thus, diagnosis of newborn infants with congenital toxoplasmosis must occur as early as possible to initiate treatment to prevent or reduce the clinical manifestations of the disease (McLeod et al. 2009).

The neonatal serologic diagnosis of congenital toxoplasmosis is based on measurement of the IgM and IgA anti-T. gondii antibodies. However, $15-30 \%$ of the children infected do not present these antibodies at birth and $10-20 \%$ of the infants testing positive for these antibodies are false-positives (Remington et al. 2004). The IgG anti-T. gondii antibodies are also indicative of congenital infection, which is known to be transmitted through the placenta from mother to child. Confirmation of congeni-

Financial support: Secretaria do Estado de Saúde de Minas Gerais, FAPEMIG (CBB-APQ-00129-09), CNPq (301110/2009-3)

+ Corresponding author: ricardovitor@icb.ufmg.br

Received 30 April 2010

Accepted 26 July 2010 tal toxoplasmosis diagnosis is currently made through observation of the persistence of IgG antibodies during the first year of life, but this procedure is lengthy, with early treatment of the infected children being necessary (Press et al. 2005).

In this study, we evaluated the use of western blot (WB) analysis in the diagnosis of neonatal congenital toxoplasmosis and its possible associations with clinical symptoms developed by the newborn child.

\section{PATIENTS, MATERIALS AND METHODS}

Patients and clinical samples - This study was carried out with infants participating in the Neonatal Screening State Program of Minas Gerais from 1 November 200631 May 2007. In this screening, dry blood samples on filter paper were collected from 146,307 newborn infants to assess IgM anti-T. gondii levels (Toxo IgM Q-Preven $\mathrm{kit}^{\circledR}$, Symbiosis, Leme, Brazil). Confirmative serologic tests were run on 227 cases with positive or undetermined screening results. These infants were tested for $\mathrm{IgG}$ and IgM anti-T. gondii [enzyme-linked fluorometric assay (ELFA-VIDAS ${ }^{\circledR}$ ), bioMérrieux SA, Lyon, France] one month after birth. Selected newborn infants were classified into two groups: (i) group 1 (infected children), which was comprised of 177 infants who tested positive for the persistence of IgG-ELFA-VIDAS ${ }^{\circledR}$ antibodies (171) or positive parasitological diagnosis by inoculation of mice (6), and (ii) group 2 (non-infected children), which was comprised of 38 infants who tested negative for IgG anti-T. gondii antibodies at the end of 12 months. Twelve infants were excluded from the study because they did not return for blood collection after 12 months for the IgG anti-T. gondii analysis. 
The children selected for this study were examined to evaluate possible macular (indirect ophthalmoscopy), neurological (transfontanel ultrasound or cranial radiography or computerized cranial tomography in selected cases) and hearing (behavioral evaluation, otoacoustic emissions and PEATE) signals, according to Andrade et al. (2008) and Vasconcelos-Santos et al. (2009). The protocols conducted in this study were approved by the local Ethical Committee (Federal University of Minas Gerais, protocol 298/06).

$W B$ - Purified tachyzoites from the RH strain of $T$. gondii $\left(10^{6}\right.$ parasites) were solubilized in sample buffer and electrophorezed on 10\% polyacrylamide gels (Mini Protean II, Bio-Rad Hercules, CA, USA) according to Vitor et al. (1999). The standards used for molecular weights $(116,97,66,45,29$ and $18 \mathrm{kDa})$ were from Sigma-Aldrich. St. Louis, MO, USA. After separation by sodium dodecyl sulfate-polyacrylamide gel electrophoresis, proteins were transferred to nitrocellulose membranes for $20 \mathrm{~h}$ at $30 \mathrm{~V}$ and $40 \mathrm{~mA}$ followed by $1 \mathrm{~h}$ at $90 \mathrm{~mA}$. Protein detection was carried out by an immunoenzymatic method. Nitrocellulose strips $(3 \mathrm{~mm})$ were incubated at room temperature with sera at a dilution of 1:100 (IgG) or 1:50 (IgM). The strips were then incubated for $1 \mathrm{~h}$ with the conjugate [anti-human IgG or anti-human IgM labelled with peroxidase (Sigma-Aldrich) diluted at 1:1000]. Paired mother and newborn serum samples were run in parallel for $\mathrm{IgG}$ analysis and newborn serum was run for IgM analysis. Bands were visualized by developing the strips using 4-chloro-1-naphthol as the substrate.

Three previously trained professionals independently observed the blots and the final result was that observed by at least two of the three. The following positivity criteria were adopted: (i) the occurrence of antigenic bands recognized by the $\mathrm{IgG}$ of the newborn infant but not of the mother, (ii) the occurrence of antigenic bands recognized more strongly by the IgG of the newborn child as compared to that of the mother (Rilling et al. 2003) and (iii) recognition of any antigenic band by the $\operatorname{IgM}$ of the newborn infant (Chumpitazi et al. 1995). Criteria (i) and (ii) allow the differentiation between maternal antibodies passively transmitted and antibodies synthesized by the infant.

Statistical analysis - A database using the software Microsoft Excel ${ }^{\circledR}$ was applied as support to the study containing confirmative IgG and IgM-ELFA-VIDAS ${ }^{\circledR}$, persistence of IgG antibodies in the newborn and the WB (IgG and IgM) results of the mother-child binome obtained in the present study. The analysis was performed using the OpenEpi version 2.3 program.

The validity of the WB tests (IgG-WB, IgM-WB and IgG-WB + IgM-WB) was estimated based on sensitivity, specificity, likelihood ratio of a positive test, likelihood ratio of a negative test and the kappa index using the results of the persistence of $\operatorname{IgG}$ antibodies at the end of 12 months as a reference standard.

The database also includes the results obtained from the clinical macular, neurological and hearing examinations of each infant. Comparisons were made by constructing 2 x 2 tables of IgG-WB, IgM-WB and IgM-ELFA-VIDAS ${ }^{\circledR}$ results and the frequencies of clinical signals in the newborn infants by using the Chi-square test, relative risks and $95 \%$ confidence interval as association measures.

\section{RESULTS}

WB for IgG and IgM antibodies - In group 1 (177 newborn infants with confirmed congenital infection), 130 tested positive by IgG-WB. Of these, 119 infants recognized at least one antigen with a different molecular weight than that recognized by the maternal serum (A in Figure). Eleven newborn infants recognized antigens similar to their mother's, but some bands had stronger intensity (B in Figure). In group 2 (38 uninfected newborn infants), 37 tested negative ( $\mathrm{C}$ in Figure).

Based on IgM-WB, 97 of the 177 infants with confirmed congenital infection tested positive (D in Figure). Among the 38 infants who did not have the infection, two presented false-positive results. When the WB results for the $\operatorname{IgG}$ and $\operatorname{IgM}$ antibodies were combined, 153 of the 177 infants infected presented positive results, while two of the 38 uninfected children presented a false-positive result.

Table I presents the sensitivity value, specificity value, likelihood ratio of a positive test, likelihood ratio of a negative test and the kappa index of the IgG-WB, IgM-WB tests and the combination of the results of the two tests.

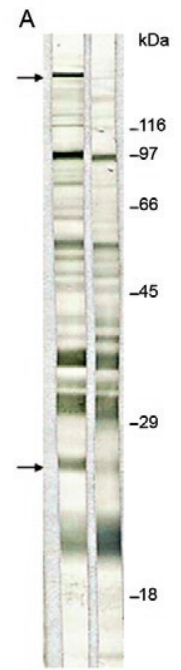

N M

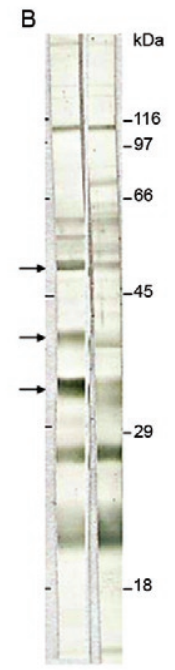

N M

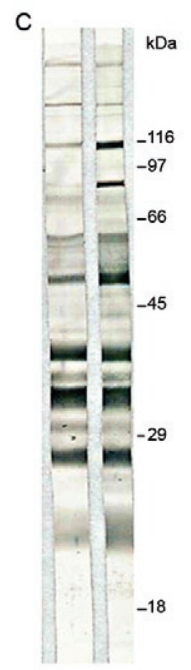

N M

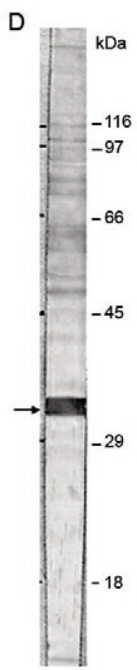

$\mathrm{N}$
Western blot analysis with paired mother and newborn sera from (A) a child congenitally infected with Toxoplasma gondii. Two antigens recognized by the child's IgG but not by the mother's IgG. B: a child congenitally infected with $T$. gondii. The intensity of three bands on the IgG blots was stronger with the infant's serum compared to the maternal serum; C: a child not infected with $T$. gondii. Antigens are similarly recognized by the child's and the mother's IgG; D: a child congenitally infected with $T$. gondii. One antigen was recognized by IgM of the infant. Arrows shows antigens recognized by the child's sera to confirm congenital toxoplasmosis. The apparent molecular weights $(\mathrm{kDa})$ of protein standards are given on the right. M: mother; $\mathrm{N}$ : newborn. 
The main antigens detected by the anti- $T$. gondii antibodies in newborn infants varied from 21-116 kDa. Three antigens with molecular weight higher than $116 \mathrm{kDa}$ were also observed, but due to the limitations of the molecular weight standards used, it was not possible to estimate their exact molecular weights. The more prevalent antigens varied from 45-66 kDa, from 97-116 $\mathrm{kDa}$ and three antigens had molecular weights above $116 \mathrm{kDa}$.

Association of the WB results and clinical diagnosis of the infants - At this stage, the results of the IgG-WB, IgM-WB and IgM-ELFA-VIDAS ${ }^{\circledR}$ tests of the newborn infants presenting confirmed toxoplasmosis (group 1) were correlated with the clinical signs observed in these infants. Table II shows the results obtained from the association of the IgG-WB, IgM-WB and IgM-ELFAVIDAS $^{\circledR}$ tests using the following parameters: macular lesions (active macular lesions or cicatricial macular lesions), neurological alterations and hearing deficiencies.
IgM-WB association in infants presenting macular lesions was statistically significant $(\mathrm{p}<0.05)$ as compared to the infants not presenting lesions. The infants presenting positive IgM-WB results had a 1.2-fold greater risk of presenting macular lesions than the children presenting negative IgM-WB results. When the macular lesion data were compared with the IgG-WB or IgM-ELFA-VIDAS ${ }^{\circledR}$ results, no significant associations were identified.

Based on these results, macular lesions were classified as active or cicatricial lesions. The association of the IgM-WB results with the presence of active macular lesions was positive $(p<0.05)$. Infants that tested positive for IgM-WB had a 1.4-fold greater risk of presenting active macular lesions as compared to those that tested negative by IgM-WB. No significant association was verified between IgG-WB or IgM-ELFA-VIDAS ${ }^{\circledR}$ results and active macular lesions. When the infants with cicatricial macular lesions were considered, no signifi-

TABLE I

Sensitivities, specificities, likelihood ratios and kappa indices of IgG-western blot (IgG-WB), IgM-WB and IgG-WB + IgM-WB for Toxoplasma gondii antibodies in newborn infants

\begin{tabular}{lccc}
\hline & IgG-WB & IgM-WB & IgG-WB + IgM-WB \\
Parameter & $(\mathrm{CI} 95 \%)$ & $(\mathrm{CI} 95 \%)$ & 86.44 \\
\hline Sensitivity \% & 73.45 & 54.8 & $(80.62-90.72)$ \\
Specificity \% & $(66.49-79.40)$ & $(47.45-61.95)$ & 94.74 \\
& 97.37 & 94.74 & $(82.71-98.54)$ \\
Positive likelihood ratio & $(86.50-99.53)$ & $(82.71-98.54)$ & 16.42 \\
Negative likelihood ratio & 27.91 & 10.41 & $(6.152-43.85)$ \\
& $(3.91-199.2)$ & $(3.843-28.21)$ & 0.1431 \\
Cohen's kappa & 0.2727 & $(0.4641-0.4904)$ & $(0.1315-0.1558)$ \\
& $(0.2612-0.2847)$ & 0.2743 & 0.6614 \\
\hline
\end{tabular}

a: parameters are given as percentages with $95 \%$ confidence interval (CI) in parentheses.

\section{TABLE II}

Association between the western blot (WB) or IgM-ELFA-VIDAS ${ }^{\circledR}$ results and the clinical signals presented by the infants with confirmed congenital toxoplasmosis

\begin{tabular}{lccccccccc}
\hline \multirow{2}{*}{$\begin{array}{l}\text { Clinical signals } \\
(\mathrm{n} / \mathrm{N})\end{array}$} & \multicolumn{3}{c}{ IgG-WB } & & \multicolumn{2}{c}{ IgM-WB } & & \multicolumn{2}{c}{ IgM - ELFA-VIDAS $^{\circledR}$} \\
\cline { 2 - 9 } & $\mathrm{RR}$ & $(95 \% \mathrm{CI})$ & $\mathrm{p}$ value & $\mathrm{RR}$ & $(95 \% \mathrm{CI})$ & $\mathrm{p}$ value & $\mathrm{RR}$ & $(95 \% \mathrm{CI})$ & $\mathrm{p}$ value \\
\hline Macular lesion (133/213) & 1.1 & $(0.9-1.3)$ & 0.49 & 1.2 & $(1.1-1.4)$ & 0.03 & 0.1 & $(0.8-1.2)$ & 0.83 \\
Active macular lesion (83/213) & 1.1 & $(0.8-1.4)$ & 0.60 & 1.4 & $(1.1-1.9)$ & 0.01 & 1.1 & $(0.8-1.7)$ & 0.54 \\
Cicatricial macular lesion (50/213) & 1.2 & $(0.7-1.9)$ & 0.46 & 1.2 & $(0.8-1.7)$ & 0.37 & 0.8 & $(0.5-1.1)$ & 0.35 \\
Neurological signals (35/207) & 1.1 & $(0.6-2.8)$ & 0.49 & 0.6 & $(0.3-1.2)$ & 0.14 & 0.5 & $(0.3-1.0)$ & 0.10 \\
Hearing deficiencies (51/134) & 0.7 & $(0.5-1.3)$ & 0.36 & 1.6 & $(0.9-3.0)$ & 0.09 & 0.9 & $(0.5-1.6)$ & 0.92 \\
\hline
\end{tabular}

CI: $95 \%$ confidence interval; $\mathrm{n}$ : number of infants with clinical signals; $\mathrm{N}$ : total number of infants submitted to clinical examinations; RR: risk relative value; WB: western blot. 
cant association was found with IgM-WB, IgG-WB or IgM-ELFA-VIDAS ${ }^{\circledR}$ results. Associations between the IgG-WB, IgM-WB or IgM-ELFA-VIDAS ${ }^{\circledR}$ tests and neurological signals or hearing deficit were not statistically significant in the infant groups with or without these clinical alterations.

\section{DISCUSSION}

In the present study, the IgG-WB reached a sensitivity of $73.5 \%$ and a specificity of $97.4 \%$. The IgMWB reached a sensitivity of $54.8 \%$ and a specificity of $94.7 \%$. Based on the values obtained in the analysis of the likelihood ratio of IgM-WB and IgG-WB (10.41 and 27.91 , respectively), the application of this test for confirmative diagnosis of neonatal toxoplasmosis is recommended. A combination of IgG-WB and IgM-WB increased the sensitivity to $86.5 \%$. A Kappa index of 0.66 shows good agreement between WB assays and those that measure the persistence of IgG antibodies.

The sensitivity and specificity of the IgG-WB and IgM-WB tests observed in this study agree with the work conducted by Pinon et al. (2001), who studied 105 infants born to mothers who had sero-converted into positivity for toxoplasmosis during pregnancy (55 infected and 50 uninfected). These authors found a sensitivity of $65.4 \%$ and a specificity of $96 \%$ in the IgG-WB test and a sensitivity of $68.5 \%$ and a specificity of $98 \%$ in the IgM-WB test. When the two tests were combined, a sensitivity of $77.7 \%$ and a specificity of $96 \%$ were observed. More recently, a commercial WB test for IgM and IgG anti-T. gondii antibodies showed a sensitivity of $80 \%$ and a specificity of $96 \%$ (Rilling et al. 2003).

Two false-positive cases occurred in this study. One infant presented a positive result both for IgG-WB and IgM-WB. This child was considered uninfected due to the absence of IgG (ELFA-VIDAS ${ }^{\circledR}$ ) seven months after birth as well as by negative serum results of the mother four months after delivery. The other child presented a false-positive IgM-WB result. The confirmative serologic examinations of this child were negative. The blood sample used for the WB tests was obtained when the child was over three months of age. Falsepositive cases have been reported in the literature when WB testing was performed on samples from children over three months of age. The authors attribute the result to the presence of natural antibodies, mostly IgM, against $T$. gondii. The origin of the antigenic stimulus leading to the production of these natural antibodies is unknown (Georges Desmonts et al. 1990, Pinon et al. 1996). In fact, Robert-Gangneux et al. (1999) carried out monthly WB tests in infants clinically suspected of being infected and found that three children who were not infected presented additional IgM bands when the test was applied after three months of age. Previously conducted WB tests presented negative results.

The most prevalent antigens in this study ranged from 45-66 kDa, from 97-116 $\mathrm{kDa}$ and above $116 \mathrm{kDa}$. Of all the antigens recognized exclusively by the children, the antigens with molecular weight above $116 \mathrm{kDa}$ corresponded to $33 \%$ of the total recognized. For 25 infants, positive diagnosis could only be con- firmed through identification of these antigens. The antigen recognition standard demonstrated in this study differs from what is commonly presented in the literature, with antigens that have molecular weights ranging from $15-100 \mathrm{kDa}$ and a predominance of antigens with molecular weights of $20 \mathrm{kDa}$ and $70 \mathrm{kDa}$. The differences observed in this study as compared to other reports (Chumpitazi et al. 1995, Robert-Gangneux et al. 1999) may be based on WB methodological differences among laboratories. Another factor to consider relates to the antigenic constitution of the T. gondii isolates found in Brazil as compared to the clonal strains predominating in the United States and Europe (Ferreira et al. 2006); differences among these strains could likely result in a differentiated antibody profile in infants with congenital toxoplasmosis in these countries.

This study investigated the association between the WB results and the clinical diagnosis of the children confirmed to be infected. The association between the IgM-WB test and the clinical diagnosis of macular lesions was significant. Infants with a positive IgM-WB result had a 1.2-fold greater chance of presenting macular lesions than those with negative IgM-WB results. The type of macular lesion was later shown to influence the association observed. An association was also found between the positive IgM-WB test and active macular lesions. The positive IgM-WB infants presented a 1.4-fold greater risk of having an active macular lesion than those testing negatively by IgM-WB. These results show that the association observed previously between $\operatorname{IgM}-\mathrm{WB}$ and macular lesions is related to the diagnosis of active macular lesions. In fact, active macular lesions are associated with an acute infection, a phase when IgM antibodies predominate. Cicatricial macular lesions are related to the chronic phase of the disease, when the IgM antibody levels are practically undetectable (Jones et al. 2003). An association between IgG-WB or IgM-ELFA-VIDAS ${ }^{\circledR}$ results and clinical diagnosis of the children (macular lesion, neurological signs and hearing deficiencies) did not show statistical significance.

This work showed that the WB assay is an effective tool for confirming the diagnosis of infants clinically suspected of congenital toxoplasmosis. The difference in the standard of antigen recognition observed in this work may indicate a differentiated profile of antibodies in response to the differential antigenic constitutions of the isolates predominating in Brazil. An association between WB results and clinical diagnosis of infants was demonstrated for the first time, with the IgM-WB test result likely being an indicator of macular lesions in newborn infants.

To our knowledge, this is the first report describing an association between WB data and clinical signs of congenital toxoplasmosis; importantly, we showed a statistically significant association between active macular lesions and the IgM-WB test.

\section{ACKNOWLEDGEMENTS}

To Rosalida Estevan Nazar Lopes, for her technical assistance. 


\section{REFERENCES}

Andrade GM, Resende LM, Goulart EM, Siqueira AL, Vitor RW, Januario JN 2008. Hearing loss in congenital toxoplasmosis detected by newborn screening. Braz J Otorhinolaryngol 74: 21-28.

Chumpitazi BF, Boussaid A, Pelloux H, Racinet C, Bost M, GoullierFleuret A 1995. Diagnosis of congenital toxoplasmosis by immunoblotting and relationship with other methods. J Clin Microbiol 33: 1479-1485

Ferreira A de M, Vitor RW, Gazzinelli RT, Melo MN 2006. Genetic analysis of natural recombinant Brazilian Toxoplasma gondii strains by multilocus PCR-RFLP. Infect Genet Evol 6: 22-31.

Georges Desmonts, Dupouy-Camet J, Lavareda De Souza S, Bougnoux ME, Mandelbrot L, Hennequin C, Dommergues M, Benarous R, Tourte-Schaefer C 1990. Preventing congenital toxoplasmosis. Lancet 336: 1017-1018.

Gilbert RE, Peckham CS 2002. Congenital toxoplasmosis in the United Kingdom: to screen or not to screen? J Med Screen 9: $135-141$

Jones J, Lopez A, Wilson M 2003. Congenital toxoplasmosis. Am Fam Physician 67: 2131-2138.

McLeod R, Kieffer F, Sautter M, Hosten T, Pelloux H 2009. Why prevent, diagnose and treat congenital toxoplasmosis? Mem Inst Oswaldo Cruz 104: 320-344.

Pinon JM, Chemla C, Villena I, Foudrinier F, Aubert D, PuygauthierToubas D, Leroux B, Dupouy D, Quereux C, Talmud M, Trenque T, Potron G, Pluot M, Remy G, Bonhomme A 1996. Early neonatal diagnosis of congenital toxoplasmosis: value of comparative enzyme-linked immunofiltration assay immunological profiles and anti-Toxoplasma gondii immunoglobulin $\mathrm{M}(\operatorname{IgM})$ or $\operatorname{Ig} \mathrm{A}$ immunocapture and implications for postnatal therapeutic strategies. J Clin Microbiol 34: 579-583.
Pinon JM, Dumon H, Chemla C, Franck J, Petersen E, Lebech M, Zufferey J, Bessieres MH, Marty P, Holliman R, Johnson J, Luyasu V, Lecolier B, Guy E, Joynson DH, Decoster A, Enders G, Pelloux H, Candolfi E 2001. Strategy for diagnosis of congenital toxoplasmosis: evaluation of methods comparing mothers and newborns and standard methods for postnatal detection of immunoglobulin G, M, and A antibodies. J Clin Microbiol 39: 2267-2271.

Press C, Montoya JG, Remington JS 2005. Use of a single serum sample for diagnosis of acute toxoplasmosis in pregnant women and other adults. J Clin Microbiol 43: 3481-3483.

Remington JS, Thulliez P, Montoya JG 2004. Recent developments for diagnosis of toxoplasmosis. J Clin Microbiol 42: 941-945.

Rilling V, Dietz K, Krczal D, Knotek F, Enders G 2003. Evaluation of a commercial $\mathrm{IgG} / \mathrm{IgM}$ western blot assay for early postnatal diagnosis of congenital toxoplasmosis. Eur J Clin Microbiol Infect Dis 22: 174-180.

Robert-Gangneux F, Gavinet MF, Ancelle T, Raymond J, TourteSchaefer C, Dupouy-Camet J 1999. Value of prenatal diagnosis and early postnatal diagnosis of congenital toxoplasmosis: retrospective study of 110 cases. J Clin Microbiol 37: 2893-2898.

Vasconcelos-Santos DV, Machado Azevedo DO, Campos WR, Oréfice F, Queiroz-Andrade GM, Carellos EV, Castro Romanelli RM, Januário JN, Resende LM, Martins-Filho OA, de Aguiar Vasconcelos Carneiro AC, Almeida Vitor RW, Caiaffa WT, UFMG Congenital Toxoplasmosis Brazilian Group 2009. Congenital toxoplasmosis in Southeastern Brazil: results of early ophthalmologic examination of a large cohort of neonates. Ophthalmology 116: 2199-2205.

Vitor RW, Ferreira AM, Fux B 1999. Antibody response in goats experimentally infected with Toxoplasma gondii. Vet Parasitol 81: 259-263. 